\title{
L'appariement forme-fonction en FL2 : le développement de l'emploi des formes verbales du passé et la primauté du marquage de l'aspect
}

\author{
Michot, Marie-Eve \& Pierrard, Michel \\ Vrije Universiteit Brussel \\ Marie-Eve.Michot@vub.ac.be \& Michel.Pierrard@vub.ac.be
}

\begin{abstract}
Résumé. Une dimension clef du processus d'acquisition d'une langue seconde (L2) est la mise en relation d'une forme et d'une fonction. Cet appariement forme-fonction ne se met néanmoins en place que fort tardivement. Ainsi, pour exprimer certaines fonctions, l'apprenant L2 a dans un premier temps recours à des principes discursifs et sémantico-pragmatiques, à des structures préfabriquées et à des formes intermédiaires avant de grammaticaliser progressivement ses énoncés et enfin d'utiliser adéquatement la grammaire pour marquer, entre autres, les différentes catégories grammaticales (nombre, genre, temps, etc.). Bref, le processus acquisitionnel passe d'un stade premier de lexicalisation vers un stade de morphologisation, qui ouvre la perspective d'un emploi adéquat, d'un appariement donc entre la forme et sa/ ses fonction(s) (Noyau 1997). En ce qui concerne l'analyse du processus d'appropriation des formes verbales, les études acquisitionnelles se sont avant tout intéressées à l'appariement entre les formes conjuguées des verbes et l'expression du temps et de l'aspect. En français, le marquage de ces deux fonctions est intrinsèquement lié. En particulier, l'exploitation émergente des conjugaisons du passé (passé composé, imparfait, passé simple) offre un champ d'observation particulièrement riche qui a suscité de nombreuses études (cf. e.a. Kaplan 1987 ; Noyau et al. 1995 ; Kihlstedt 2002 ; Ayoun 2001, 2004, 2005). Plusieurs études ont ainsi indiqué que les formes de la conjugaison du passé se limitent dans un premier stade au passé composé qui est par conséquent souvent suremployé à des contextes où devraient apparaître des formes de l'imparfait. L'imparfait s'impose ensuite progressivement, d'abord avec les verbes être et avoir et plus tard avec les autres verbes lexicaux. Le plus-que-parfait est la conjugaison du passé qui surgit en dernier lieu dans l'interlangue des apprenants de français L2 (FL2). La contribution se donne dans un premier temps pour but de décrire le développement des formes de la conjugaison du passé dans l'interlangue d'apprenants néerlandophones du FL2 à l'aide d'un corpus oral. Elle examinera ensuite les fonctions temporelle et/ ou aspectuelle que ces formes verbales y remplissent afin d'étudier leur appariement forme-fonction. La contribution tentera plus particulièrement de répondre aux questions suivantes, issues de l'hypothèse aspectuelle d'Andersen (1986, 1991) : L'aspect est-il effectivement marqué avant le temps en FL2 ? L'imperfectif apparaît-il après le perfectif en FL2 ? Les apprenants marquent-ils d'abord le perfectif avec des verbes téliques, et seulement ensuite éventuellement avec des verbes atéliques ? L'imperfectif est-il d'abord utilisé avec des verbes atéliques et seulement après avec des verbes téliques? Les données de notre corpus confirment prudemment l'importance et la primauté du marquage de l'aspect dans l'interlangue des apprenants FL2. Cette primauté de l'aspect permet sans doute aussi de comprendre la primauté des formes du passé composé, qui s'opposent par l'aspect accompli au présent qui indique l'accomplissement. Le surgissement de l'imparfait semble aller de pair avec la prise en compte de l'expression du temps passé par le passé composé et donc aussi de la nécessité d'introduire une dimension d'accomplissement au passé. L'analyse détaillée des lexèmes verbaux convoqués indique que leur aspect lexical inhérent semble aussi jouer un rôle dans l'ordre développemental des conjuguaisons du passé, bien que certaines affirmations de l'hypothèse aspectuelle d'Andersen n'aient pas pu être pleinement confirmées.
\end{abstract}

\begin{abstract}
A key dimension of the acquisiton process of a second language (L2) is the link between a form and it's function, but this form-function pairing emerges rather lately. In order to express certain functions, the L2 learner first uses semantico-pragmatic resources, chunks and intermediate forms before being able to progressively grammaticalise his utterances and finally to adequately use the grammar to mark different grammatical categories (e.g. number, gender, tense, etc.). The $\mathrm{L} 2$ acquisition process evolves thus from a lexicalisation to a morphologisation stage, which progressively enables the L2 learner to adequately combine form and function (Noyau 1997). Regarding the acquisition process of verbs, most studies have examined the pairing of finite verbs and the expression of tense (temps in French) and aspect (aspect). The marking of these two functions is intrinsically linked in French. The progressive exploitation of the conjugations of the past (passé composé, imparfait, passé simple) offers in particular a rich investigation field (cf. i.a. Kaplan 1987; Noyau et al. 1995; Kihlstedt 2002; Ayoun 2001, 2004, 2005). Several studies have revealed that the reference to the past is limited to the passé composé at first, which is consequently overused, even in contexts where the imparfait should appear. The imparfait develops progressively afterwards, in the first place with the verbs être and avoir and later with the other
\end{abstract}


lexical verbs. The plus-que-parfait is the last conjugation of the past to develop in the interlanguage of FL2 learners. This contribution will first describe the development of the different conjugations of the past in the interlanguage of Dutch speaking FL2 learners, by examining oral data. The focus of this contribution is the temporal and/ or aspectual functions expressed by these conjugations. This paper will more particularly enable us to answer the following research questions, that are derived from Andersen's Aspect Hypothesis (Andersen 1986, 1991):Is aspect actually marked before tense in FL2? Does the imperfective emerge after the perfective in FL2? Is the perfective first marked with telic verbs and afterwards marked with atelic verbs? Is the imperfective first used with atelic verbs and only afterwards used with telic verbs? The data of our corpus cautiously confirm the primacy of aspectual marking in the interlanguage of FL2 learners. This primacy of aspect seems to explain the primacy of the passé composé forms, since they express the perfective in opposition to the present tense which expresses the imperfective. The later emergence of the imparfait seems to go hand in hand with the actual reference to the past with passé composé forms and therefore with the necessity to introduce an imperfective dimension in the past. The detailed analysis of the produced verbs indicates that the inherent lexica aspect also plays a role in the developmental order of the conjugations of the past, even though certain elements of Andersen's Aspect Hypothesis have not been fully confirmed by the data.

Une dimension clef du processus d'acquisition d'une langue seconde (L2) est la mise en relation d'une forme et d'une fonction. Selon Véronique (2009 : 302), l'apprenant L2 cherche la forme adéquate pour exprimer une certaine fonction qui lui est déjà connue grâce à son expérience langagière antérieure. La conjonction adéquate d'une forme et d'une fonction constitue cependant l'aboutissement d'un long procès d'appropriation. En effet, forme et fonction ne sont pas indissociablement liées dès le début du processus développemental (Perdue \& Klein 1992 ; Perdue et al. 2002). Ainsi, l'absence de marques morphologiques n'indique pas automatiquement une absence d'indications fonctionnelles dans l'interlangue de l'apprenant (p. ex. l'emploi d'adverbes temporels peut compenser l'absence de morphologie verbale pour marquer des nuances temporelles). De même, la présence d'une forme morphologique n'implique pas nécessairement la maîtrise de la fonction ou de l'une des fonctions qui lui est attribuée dans la langue cible (p. ex. l’emploi précoce de structures préfabriquées ou « chunks », alors que le système flexionnel n’est pas encore développé ou activé).

L'appariement forme-fonction d'une L2 ne se met en place que fort tardivement. Ainsi, pour exprimer certaines fonctions, l'apprenant L2 a dans un premier temps recours à des principes discursifs et sémantico-pragmatiques, à des structures préfabriquées et à des formes intermédiaires avant de grammaticaliser progressivement ses énoncés et enfin d'utiliser adéquatement la grammaire pour marquer, entre autres, les différentes catégories grammaticales (nombre, genre, temps, etc.). Bref, le processus acquisitionnel passe d'un stade premier de lexicalisation vers un stade de morphologisation, qui ouvre la perspective d'un emploi adéquat, d'un appariement donc entre la forme et sa/ ses fonction(s) (Noyau 1997 ; Ullman 2001).

En ce qui concerne l'analyse du processus d'appropriation des formes verbales, les études acquisitionnelles se sont avant tout intéressées à l'appariement entre les formes conjuguées des verbes et l'expression du temps et de l'aspect. En français, le marquage de ces deux fonctions est intrinsèquement lié. En particulier, l'exploitation émergente des conjugaisons du passé (passé composé, imparfait, passé simple) offre un champ d'observation particulièrement riche qui a suscité de nombreuses études (cf. e.a. Kaplan 1987 ; Noyau et al. 1995 ; Kihlstedt 2002 ; Ayoun 2001, 2004, 2005). Bartning et Schlyter (2004) ont proposé une description de l'itinéraire acquisitionnel d'apprenants suéodophones du français langue seconde (FL2) en balisant les différents stades, entre autres, par le développement de la morphologie verbale TAM (temps, aspect, mode) à l'oral sous trois volets : l'expression du passé, du futur et l'emploi du subjonctif. L'étude de Schlyter (2003) y a également inclus l'emploi des coverbes ${ }^{1}$ de modalité. L’itinéraire acquisitionnel des conjugaisons du passé correspond à celui observé dans d'autres études sur le marquage temporel en français L2 (Kaplan 1987 ; Kihlstedt 1998 ; Howard 2002). Dans un premier stade, les formes de la conjugaison du passé se limitent au passé composé qui est par conséquent souvent suremployé à des contextes où devraient apparaître des formes de l'imparfait. L'imparfait s'impose ensuite progressivement, d'abord avec les verbes être et avoir et plus tard avec les autres verbes lexicaux. Le plus-que-parfait est la conjugaison du passé qui surgit en dernier lieu dans l'interlangue des apprenants FL2. 
La présente contribution a pour objectif de décrire le processus d'appropriation de la morphologie temporo-aspectuelle en FL2 d'apprenants néerlandophones, et plus particulièrement d'examiner l'emploi aspectuel et/ ou temporel des diverses formes verbales conjuguées au passé composé, à l'imparfait ou au plus-que-parfait.

\section{Approches théoriques et méthodologiques}

Selon Véronique (2009), le processus d'appariement forme-fonction au niveau des formes verbales en FL2 privilégie, dans une première phase, le marquage de l'aspect. Il confirmerait donc pour le français langue seconde la pertinence de l'hypothèse aspectuelle d'Andersen (1986, 1991). Nous présenterons d'abord d'abord nos définitions succinctes du temps et de l'aspect avant d'esquisser les thèses principales d'Andersen et de formuler nos questions de recherche.

\subsection{Définition du temps et de l'aspect}

Le temps et l'aspect sont intrinsèquement liés en français. Une définition générale des deux notions est présentée ci-dessous.

\subsubsection{Le temps}

Le temps linguistique est exprimé par une multitude de mots : noms, adjectifs, adverbes, conjonctions, prépositions. Selon Wilmet (2010 : 176), l’unique mot temporel spécialisé est cependant le verbe. Le temps verbal permet de rapporter un procès à un point quelconque du temps cosmique (un repère $\mathrm{R}$ ). Il peut y être concomitant (1), antérieur (2) ou postérieur (3).

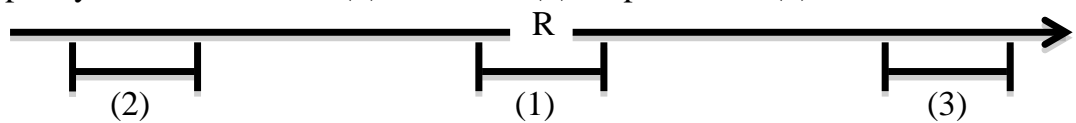

En intégrant au point $R$ la dimension supplémentaire du maintenant, un nouveau repère cosmique est créé : l’actualité (A). Ce repère permet de répartir le procès dans trois époques : le présent, le passé et le futur.

$\longrightarrow$ passé $\quad$ présent futur

Cette définition de temps, proposée par Wilmet, est basée sur le système tripartite de Reichenbach (1947) qui distingue le point de l'énonciation, le point de l'événement et le point de référence. Starren (2001 : 18) signale que ce système, bien que souvent employé dans des études acquisitionnelles, présente plusieurs défauts. La première critique de Starren porte sur la distinction de points temporels au lieu d'intervalles temporels (time spans) : "time points cannot include each other whereas the difference between the aspectual notions imperfectivity and perfectivity have to be explained in terms of time spans which can include each other" (Starren 2001 : 18). Cette remarque ne concerne pas vraiment le temps, mais plutôt l'aspect et sera donc discutée sous le prochain point. Un autre problème du système de Reichenbach, signalé par Starren, touche à la définition de la notion de point de référence. Elle est trop vague et peut être interprétée de différentes façons : le point de référence est un événement décrit dans le contexte, il est donné au moyen d'un adverbe temporel, il est donné par une proposition subordonnée, etc. (Starren 2001 : 18).

En raison de ces défauts, Starren préfère un autre système pour définir le concept de temps (et celui d'aspect), le Basic Time Structure de Klein (1994, 1995). Ce système distingue les trois paramètres suivants : (a) le temps topique (TT), (b) le temps de l'énonciation (TU - time of utterance) et (c) le temps de la situation (TSit). Le TT est le temps pour lequel l'énoncé fait une assertion (Klein 1995 : 23). Il 
permet d'exprimer les relations temporelles/ les propriétés temporelles externes, c'est-à-dire de situer l'énoncé par rapport au TU sur l'axe temporel. Il peut être antérieur au TU et situer l'énoncé dans le passé, postérieur au TU et le situer dans le futur ou il peut coïncider avec le TU et ainsi exprimer le présent. Le TSit fait référence au temps de l'action même et permet de rendre compte des relations temporelles internes - l'aspect - en se situant par rapport au TT.

\subsubsection{L'aspect}

La définition de l'aspect que nous proposons est tirée de l'ouvrage de Wilmet (2010), qui s'inspire de Guillaume (1964) en distinguant le temps impliqué du temps expliqué. Le temps impliqué correspond au temps que le verbe retient en soi par définition et le temps expliqué au temps divisible en moments distincts que le discours lui attribue (passé, présent et futur) (Wilmet 2010 : 182-183). Le temps impliqué concorde avec l'aspect et le temps expliqué avec le temps comme nous l'avons défini (cf. supra).

L'aspect permet donc d'envisager le procès en lui-même, d'un point de vue interne, allant d'un terminus a quo $(\alpha)$ à un terminus ad quem $(\omega)$ et réfère à « la totalité des informations touchant le pôle A (avec ses antécédents liés < A), le pôle $\Omega$ (avec ses conséquents liés $>\Omega$ ) et l'intervalle A- $\Omega$ » (Wilmet 2010 : 184). Le procès est donc saisi à différents stades de sa réalisation, du stade antérieur au début du procès au stade postérieur à son terme final (Riegel et al. 2009 : 451).

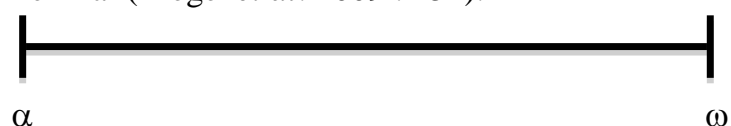

Deux types d'aspect sont distingués : l'aspect lexical et l'aspect grammatical. L'aspect lexical permet de catégoriser les verbes selon leur sémantisme interne. Vendler $(1957,1967)$ distingue ainsi quatre classes sémantiques :

a) Les verbes d'activité qui sont (i) dynamiques, (ii) atéliques et (iii) duratifs. Ces verbes expriment une activité demandant un effort de la part de l'agent (i), leur procès inhérent n’a pas de point final (ii) et il prend un certain temps (iii). Ex. courir, regarder la télévision, se promener, etc.

b) Les verbes d'accomplissement sont également dynamiques et duratifs, mais ils sont téliques car leur procès inhérent a un point final. Ex. persuader, traverser, écrire une lettre, etc.

c) Les verbes d'achèvement sont également téliques, mais ils ne sont ni dynamiques, ni duratifs. Les verbes d'accomplissement réfèrent à des périodes précises et uniques, alors que les verbes d'achèvement réfèrent à des instants précis et uniques, ponctuels. Il est ainsi possible de traverser un champ pendant des heures, ce qui n'est pas possible pour le fait de remarquer quelqu'un ou quelque chose. Ex. reconnaître, remarquer, apercevoir, etc.

d) Les verbes d'état, enfin, qui ne sont pas dynamiques ; l'état exprimé par le verbe n’est pas le résultat d'efforts faits par l'agent et ils sont atéliques et duratifs. Ex. savoir, aimer, etc.

L'aspect grammatical décrit la position du repère par rapport au procès $\alpha-\omega$ et peut être exprimé par plusieurs moyens linguistiques distincts (Riegel et al. 2009 : 519-524 ; Wilmet 2010 : 187-189) :

- le verbe et la forme verbale conjuguée : l'emploi d'une forme composée ou d'une forme simple (aspect perfectif vs imperfectif), le temps (aspect sécant vs non-sécant)

- les périphrases verbales et les coverbes suivis de l'infinitif

- les procédés de formation du vocabulaire : préfixes (re-), infixes (-aill-, -ass-, -el-, -ill-, -in-, etc.), forme pronominale (aspect inchoatif/ itératif/ progressif)

- les compléments circonstanciels (aspect semelfactif vs itératif) 
Il est important de remarquer que les conjugaisons du français, en particulier celles du passé, peuvent servir à exprimer tant le temps que le passé. Nous illustrons ce dernier point à l'aide des exemples suivants :

- $\quad$ En 1492, C. Colomb a découvert l’Amérique (marque la temporalité)

- Quel malheur, bébé est tombé ! (marque l’aspect accompli)

\subsection{L'hypothèse aspectuelle}

Diverses hypothèses ont été avancées pour interpréter l'apparition progressive de la morphologie temporo-aspectuelle chez les apprenants L2: l'hypothèse aspectuelle (Andersen 1986, 1991), l'hypothèse discursive (Bardovi-Harlig 1994, 2000) et l’hypothèse du temps passé par défaut (Salaberry 1999). Nous nous centrerons dans cette contribution sur les différents volets de l'hypothèse aspectuelle afin d'en évaluer le fonctionnement à partir d'un corpus d'apprenants FL2 de différents niveaux.

Selon l'hypothèse aspectuelle d'Andersen (1986, 1991), l'apprenant L2 utilise dans un premier temps la flexion verbale pour rendre compte de l'aspect avant de l'exploiter pour indiquer le temps. Plus précisément, Andersen affirme que le marquage de l'aspect lexical inhérent des verbes précède le marquage de l'aspect grammatical ou du temps (Andersen 1991 : 307, 319). L'hypothèse aspectuelle peut ensuite être décomposée en quatre affirmations (Li \& Shirai $2000: 50$ ) :

1. Quand une langue distingue les aspects perfectif/imperfectif morphologiquement, l'imperfectif apparaît après le perfectif.

2. Les apprenants marquent le perfectif premièrement sur des verbes d'achèvement et d'accomplissement (c'est-à-dire des verbes téliques), ensuite éventuellement sur des verbes d'activité et des verbes d'état (verbes atéliques).

3. Les premières distinctions imperfectives sont marquées sur des verbes d'état et des verbes d'activité et seulement par après sur des verbes d'accomplissement et d'achèvement.

4. Pour les langues exprimant l'aspect progressif, le marquage du progressif se fait d'abord avec les verbes d'activité et s'étend ensuite aux verbes d'accomplissement et d'achèvement, mais est rarement étendu aux verbes d'état.

La contribution se donne pour but de décrire le développement des formes verbales du passé dans l'interlangue d'apprenants néerlandophones du FL2 et d'examiner les fonctions temporelle et/ ou aspectuelle qu'elles y remplissent afin d'étudier leur appariement forme-fonction.

\subsection{Corpus}

L'étude examine l'évolution de l'acquisition de la morphologie temporo-aspectuelle à l'aide d'un corpus oral composé de trois groupes d'apprenants néerlandophones suivant des cours de FL2 dans l'enseignement secondaire flamand (partie néerlandophone de la Belgique). Ces groupes se situent à des niveaux scolaires différents et présentent un nombre d'heures d'enseignement de FL2 croissant (cf. tableau 1). Ils ont raconté l'histoire de la grenouille (Mayer 1969), une tâche orale semi-spontanée couramment utilisée dans les études acquisitionnelles.

Tableau 1 : Présentation du corpus

\begin{tabular}{|l|c|c|}
\cline { 2 - 3 } \multicolumn{1}{c|}{} & \# d'apprenants & \# d'heures de FL2 \\
\hline NN1 & 30 & \pm 250 \\
\hline NN3 & 25 & \pm 520 \\
\hline
\end{tabular}

Le groupe NN1 est composé de 30 élèves de première année de secondaire (âgés de 12-13 ans) ayant suivi environ 250 heures de FL2, le groupe NN3 représente 25 élèves (14-15 ans) de troisième année 
ayant suivi environ 520 heures de FL2 et le troisième groupe, NN6, est composé de 32 élèves (17-18 ans) de la dernière année du secondaire qui ont suivi environ 710 heures de FL2. L'avantage du corpus utilisé est qu'il permet d'évaluer le développement de l'accord sujet-verbe d'apprenants ayant acquis le FL2 dans un même contexte éducatif et linguistique, avec des approches pédagogiques, un programme éducatif et des objectifs finaux imposés par le ministère de l’enseignement.

Les productions orales furent enregistrées et ensuite transcrites selon les conventions CHAT, les données recueillies furent traitées à l'aide du logiciel CLAN (The CHILDES Project, MacWhinney 2000).

Le corpus permet de décrire le processus d'appropriation des formes verbales et d'analyser l'emploi aspectuel et/ ou temporel des diverses formes verbales conjuguées au passé composé, à l’imparfait ou au plus-que-parfait.

\subsection{Hypothèses de recherche}

Après avoir présenté une description de l'itinéraire développemental des formes verbales du passé des apprenants néerlandophones, la présente contribution tentera de répondre aux questions suivantes, issues de l'hypothèse aspectuelle d'Andersen :

1. L'aspect est-il effectivement marqué avant le temps en FL2 ?

2. L’imperfectif apparaît-il après le perfectif en FL2 ?

3. Les apprenants marquent-ils d'abord le perfectif avec des verbes téliques, et seulement ensuite éventuellement avec des verbes atéliques ?

4. L’imperfectif est-il d'abord utilisé avec des verbes atéliques et seulement après avec des verbes téliques?

\section{Description et analyse des données}

Nous présenterons d'abord le développement des différentes conjugaisons du passé dans la production des apprenants des trois groupes du corpus (§2.1), cela nous permettra ensuite de répondre plus précisément aux quatre questions de recherche (§2.2).

\subsection{Développement des formes verbales du passé}

Le tableau 2 contient les données relatives aux conjugaisons du passé, incluant l'emploi du passé composé, de l’imparfait et du plus-que-parfait.

Tableau 2. Développement des conjugaisons du passé

\begin{tabular}{|l|c|c|c|c|c|c|}
\cline { 2 - 7 } \multicolumn{1}{c|}{} & \multicolumn{2}{c|}{ NN1 } & \multicolumn{2}{c|}{ NN3 } & \multicolumn{2}{c|}{ NN6 } \\
\cline { 2 - 7 } \multicolumn{1}{c|}{} & $\#$ & $\%^{\text {totV }}$ & $\#$ & $\%^{\text {totV }}$ & $\#$ & ${ }^{\text {totV }}$ \\
\hline passé composé & 7 & 0,02 & 35 & 0,06 & 86 & 0,07 \\
\hline imparfait & $/$ & $/$ & 9 & 0,01 & 23 & 0,02 \\
\hline plus-que-parfait & $/$ & $/$ & $/$ & $/$ & 11 & 0,01 \\
\hline Total passé & $\mathbf{7}$ & $\mathbf{0 , 0 2}$ & $\mathbf{4 4}$ & $\mathbf{0 , 0 7}$ & $\mathbf{1 2 0}$ & $\mathbf{0 , 1 0}$ \\
\hline
\end{tabular}

\# : $\quad$ fréquence absolue

$\%^{\text {totV }}$ : fréquence relative par rapport au nombre total de formes verbales produites

Les trois conjugaisons du passé se développent progressivement, passant de $2 \%$ des formes verbales produites à $10 \%$ dans le groupe NN6. Dans le corpus NN1, elles se concrétisent uniquement sous la forme du passé composé. Les sept occurrences représentent $2 \%$ du nombre total de formes verbales produites. L’imparfait apparaît timidement dans le corpus NN3, apparition qui va de pair avec un emploi plus productif du passé composé $\left(6 \%^{\text {totV }}\right)$. L’emploi de la morphologie du passé est plus général et plus 
riche dans le corpus NN6, d'une part par l'emploi plus productif de l'imparfait, mais également par l'apparition de quelques occurrences au plus-que-parfait.

Tableau 3. Répartition des occurrences du passé composé et de l’imparfait

\begin{tabular}{|l|c|c|c|c|c|c|}
\multicolumn{1}{c|}{} & \multicolumn{3}{c|}{ Passé composé } & \multicolumn{3}{c|}{ Imparfait } \\
\cline { 2 - 9 } \multicolumn{1}{c|}{} & NN1 & NN3 & NN6 & NN1 & NN3 & NN6 \\
\hline \# formes & 7 & 35 & 86 & 0 & 9 & 23 \\
\hline M apprenants produisant formes & $17 \%$ & $68 \%$ & $94 \%$ & $/$ & $20 \%$ & $31 \%$ \\
\hline Écart-type & 0,2 & 1,4 & 2,7 & $/$ & 0,4 & 0,7 \\
\hline \# min. formes & 0.63 & 1.5 & 2.13 & $/$ & 0.86 & 1.55 \\
\hline \# max. formes & 0 & 0 & 0 & $/$ & 0 & 0 \\
\hline
\end{tabular}

Le pourcentage d'apprenants produisant des formes conjuguées au passé composé et à l'imparfait augmente, ainsi que le nombre de formes produites par narration. La tendance est beaucoup plus nette pour le passé composé que pour l'imparfait, ce qui s'explique par le fait que le processus s'amorce bien plus tôt dans le premier cas. Ainsi, alors qu'une minorité d'apprenants utilise l'imparfait au sein du groupe le plus avancé, la quasi-totalité de ceux-ci produit des occurrences du passé composé.

Les valeurs de l'écart-type sont très basses et relativement stables, augmentant très légèrement pour l'emploi du passé composé et indiquant donc que ces données sont un peu plus dispersées dans le corpus NN6 que dans le corpus NN3. La grande majorité des apprenants NN1 (80\%) et presque la moitié des apprenants du groupe NN3 (47\%) qui produisent des occurrences du passé composé n'en produisent qu'une seule, mais dès NN6, 60\% des apprenants en produisent au moins deux. Par contre 60\% des utilisateurs de l'imparfait en NN6 ne l'emploient toujours qu'une seule fois. Bref, l'extension progressive de l'emploi des conjugaisons du passé est nette au sein des groupes et cela tend à augmenter légèrement la différenciation entre apprenants, ce qui transparaît particulièrement au sein du groupe NN6.

\subsection{Confrontation des données receuillies aux hypothèses}

\subsubsection{L'aspect est marqué avant le temps en FL2}

La première analyse des données a permis de relever que l'emploi des formes verbales du passé est limité au passé composé chez les apprenants les moins avancés du groupe NN1. En effet, l'imparfait n'apparaît qu'à partir du corpus NN3. L'analyse détaillée des productions permet de constater que les apprenants ont tendance à décrire ce qu'ils voient sur les images qui leur sont présentées, plutôt que de raconter une histoire inspirée par les images. Cette tendance à la description plutôt qu'à la narration est plus particulièrement présente chez les apprenants du groupe NN1.

Le premier volet de l'hypothèse aspectuelle d'Andersen $(1986,1991)$ postule que les apprenants L2 utilisent dans un premier temps la flexion verbale pour rendre compte de l'aspect avant de l'exploiter pour indiquer le temps. Pour évaluer la fonction temporelle ou aspectuelle des formes verbales du passé dans notre corpus, nous utiliserons les critères suivants :

- la présence dans le récit de marques signalant une chronologie, une nuance temporelle (indices de rapports d’antériorité, de postériorité, voire de simultanéité) ;

- l'alternance entre formes verbales simples et formes verbales composées, indiquant une alternance accomplissement (imperfectif) vs accompli (perfectif) ;

- le caractère télique du verbe conjugué au passé composé (aspect lexical, cf. §1.1.2.).

(a) groupe NN1 : sept occurrences du passé composé sont produites par ces apprenants. Les sept passés composés sont indéniablement produits dans des contextes descriptifs situés dans le présent. Aucune occurrence n'est d'ailleurs accompagnée d'un marqueur temporel. Néanmoins, plusieurs occurrences 
semblent marquer une nuance aspectuelle. La nuance aspectuelle est ainsi marquée dans deux énoncés par l’opposition d'une forme verbale simple et d'une forme verbale composée (cf. ex. 1 et 2).

(1) NN1-4 : *PUP: un petit garçon regarde \#\#\# à [*] la euh@i gre@il [*]

*INT: grenouille

*PUP: grenouille

*PUP: le petit garçon va [/] va dormir

*INT: oui

*PUP: euh@i mais le [*] grenouille va partir \#\#\#

*PUP: le matin le [/] le petit garçon \# voit le [*] grenouille est \# partir [*]

(2) NN1-227 : *PUP: le garçon il est [//] \# fait son <\&kleren \&zijn \&kleren> [= vêtements ses vêtements]

*PUP: il \#

*INT: et qu'est ce qui se passe ici?

*PUP: <\&hij \&roept \&die \&kikker> [= il appelle cette grenouille]

*INT: et le chien?

*PUP: <ils sont> [//] \#\# ils voient euh@i le [*] grenouille est parti

*PUP: le garçon il est [//] \# fait son <\&kleren \&zijn \&kleren> [= vêtements ses vêtements]

Dans le cas de trois autres occurrences, l'interprétation aspectuelle est favorisée par les propriétés mêmes du lexème verbal, qui a un caractère télique très marqué (cf. ex. 3,4 ), avec parfois une opposition nette non télique/ télique (5) :

(3) NN1-227 : *PUP: un petit garçon et \# son chien euh@i \#\# @comment: wat is kikker [= c'est quoi grenouille]? *PUP: euh@i \# ils [/] ils ont un [*]

*INT: grenouille

*PUP: grenouille

*PUP: et le petit garçon et son chien dormir [*]

*PUP: euh@i le \#

*INT: grenouille

*PUP: grenouille euh@i il [*] $\underline{\text { est parti }}$

(4) NN1-227 : *PUP: le garçon il est [//] \# fait son <\&kleren \&zijn \&kleren> [= vêtements ses vêtements]

*PUP: il \#

*INT: et qu'est ce qui se passe ici?

*PUP: <\&hij \&roept \&die \&kikker> [= il appelle cette grenouille]

*INT: et le chien?

*PUP: il est tombE

*PUP: le petit garçon il [*] euh@i

*PUP: il pakt@nld[= prend] [*] le chien

*PUP: euh@i <\&ze \&roepen \&die> [= ils appellent ce]

*PUP: le petit garçon et le chien euh@i ils roepen@nld[= crier]

*INT: ils crient

*PUP: ils crient [*] le [*] grenouille

(5) NN1-141 : *PUP: et l’animal marchE [*] jusqu'à euh@i afgrond@nld[= un ravin]

*INT: un ravin

*PUP: un ravin

*PUP: et <le animal $>$ [//] l'animal arrête

*PUP: mais le garçon et le chien tombent dans le [/] le ravin

*PUP: et la [*] ravin <\&denk \&ik>@nld[= je pense]

*INT: un

*PUP: dans le ravin

*PUP: et [/] euh@i et il tombe dans l(a) eau

*PUP: puis il écoute les grenouilles

*PUP: il [/] il vait cherchE

*PUP: et [/] et [/] \#\# et quoi [*] il habite

*PUP: un [*] famil@il [*] de grenouilles

*PUP: et la grenouille euh@i

*PUP: la grenouille 
*PUP: que cherchE [//] euh@i ils cherchE [//] euh@i ont trouvE

*PUP: et puis il [*] content

Il est plus difficile d'avancer une argumentation permettant de favoriser une des deux interprétations fonctionnelles dans le cas des deux dernières occurrences. Les contextes ne révèlent pas d'indices pour l'emploi temporel ou aspectuel des passés composés. L'analyse est entravée par l'emploi d'un lexème verbal erroné par les apprenants, qui ont probablement voulu utiliser les verbes chercher et crier. Ces passés composés tendent toutefois à s'inscrire dans la description d'actions accomplies par le garçon et son chien. Une quelconque valeur aspectuelle est incertaine, mais l'expression du passé semble exclue :

(6) NN1-176 : *PUP: euh@i le garçon et le chien euh@i kijken@nld[= regarder]

*INT: regardent

*PUP: regardent le [*] grenouille

*PUP: le garçon et le chien euh@i dormir

*PUP: le [*] nuit euh@i \#\#\#

*INT: la grenouille s'échappe

*PUP: la grenouille s'échappe

*PUP: le chien et le garçon regardE [*] la boite

*PUP: euh@i et le [*] grenouille est ne pas [*] dans le [*] boîte

*PUP: le chien et le garçon $\underline{\text { a regardE }}$ [*] pour [*] le [*] grenouille

(7) NN1-128 : *PUP: en@nld[//] euh@i et euh@i l’enfant regarde dans [/] euh@i dans le [*] chaussure

*PUP: et le chien dans

*INT: le bocal

*PUP: le bocal

*PUP: l'enfant euh@i chantE [*]

*INT: wablief@nld [= pardon]?

*PUP: l'enfant $\underline{\text { a chantE }}$

(b) groupe NN3 : la fréquence des formes verbales du passé se développe, tant dans la production du passé composé, que par l'apparition des premières formes de l'imparfait. L'appariement des formes à une fonction spécifique confirme les tendances relevées pour l’emploi des passés composés dans le corpus NN1. Les nuances aspectuelles sont régulièrement marquées par l'opposition de formes verbales simples et de formes verbales composées (cf. ex. 8) ou par la télécité du lexème verbal même (ex. 9)

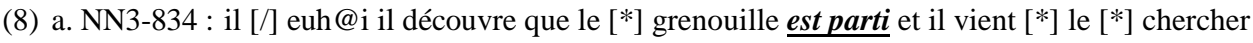

b. NN3-611 : et le garçon euh@i regarde [*] euh@i l'animal est fini [*]

(9) a. NN3-610 : *INT: que se passe-t-il?

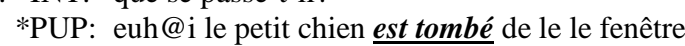

b. NN3-613 : *INT: où est le garçon maintenant?

*PUP: <hij valt>@nld [= il tombe] [//] il est tombé dans le hert

Cependant, les apprenants NN3 produisent également les premières occurrences de passé composé où une fonction temporelle semble être activée. La nuance temporelle paraît incontestable dans 10a, étant donné que l'apprenant présente sa production comme une narration et que la première illustration que l'apprenant reçoit est postérieure à l'action décrite. Les emplois sous 10b et 10c n'excluent pas la fonction temporelle des passés composés, mais l'appariement forme-fonction y semble moins transparent que dans 10a.

(10) a. NN3-834 : *PUP: euh@i [/] euh@i l'histoire va [*] euh@i un garçon \#\# euh@i

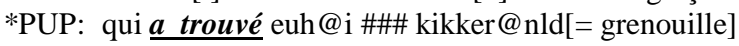

b. NN3-605 : *PUP: euh@i \#\# il y a un garçon

*PUP: euh@i il a un chien

*PUP: et il a attrapé une euh@i grenouille

*PUP: et il a euh@i placé le [*] dans une [*] bocal

c. NN3-793 : *PUP : euhm il y a une un jeu jeune garçon

*PUP : qui l'a trouvE une grenouille 
L’adéquation fonctionnelle dans l'emploi des formes du passé composé est toutefois encore loin d'être complète :

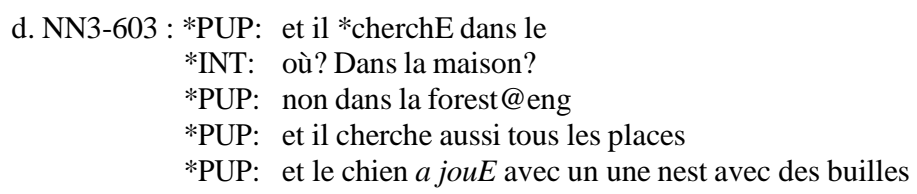

Neuf occurrences de l'imparfait sont également produites. Quelques imparfaits semblent témoigner d'un emploi temporel (ex. 11). L'ancrage temporel de la forme verbale est renforcé par l'emploi de marqueurs temporels dans 11a et 11b et l'action à l'imparfait dans 11c est clairement antérieure à la forme verbale au présent dans la narration.

(11) a. NN3-605 : *PUP: et euh@i \#\# pendant quelques minutes euh@i ils vont euh@i dehors [//] chercher dehors

*PUP: parce que le [*] fenêtre euh@i était euh@i ouvert [*]

b. NN3-605 : *PUP: ils cherchent

*PUP: et ils vont dans le bois

*PUP: euh@i \# encore euh@i quel [//] un peu plus tard il euh@i \#\#<oei wat voor beest is dat?>@nld[= c’est quel animal?] euh@i \#\#\# il était attaqué par un uil@nld [= hibou]

c. NN3-605 : *PUP: et euh@i derrière une pièce de [/] de [/] de bois il trouve euh@i ce [*] grenouille qu'il était euh@i cherché euh@i pour ja@nld [= oui]

L’appariement forme-fonction ne semble pas encore maîtrisé dans les autres énoncés (ex. 12). Les premières occurrences de l'imparfait indiquent que les apprenants FL2 commencent à utiliser les formes (ou au moins les suffixes de l'imparfait, comme illustré dans $12 \mathrm{f}$ où l'apprenant joint le suffixe de l’imparfait à un radical erronné), mais sans nécessairement maîtriser sa fonction.

(12) a. NN3-603 : *PUP: et demain [*] le boomstam il fait des deux grenouilles

*PUP: le grenouille

*PUP: qui a fait avec euh@i le garçon et des petits grenouilles

*PUP: et il il avait un petit grenouille avec elle

b. NN3-606 : *PUP: euh@i c'est un garçon avec son chien \#

*PUP: et on [*] regardait [*] d'[*] un [*] grenouille

*PUP: le chien euh@i et le garçon va [/] \#\# euh@i \#\#\# va [*] dormir

c. NN3-790 : PUP: et il vient $[*]$ dans $[/]<$ dans un $>[/]$ dans un $[/]$ un arbre

*PUP: et euh@i \# ils sont des [/] des abeilles

*PUP: en@nld [= et] [//] euh@i et il [/] il cherchait [*] dans [/] dans le

d. NN3-790 : *INT: et qu'est ce qu'il fait là sa petite montagne?

*PUP: il [/] euh@i il [/] il cherchait [*] pour [*] la grenouille et \#+//

e. NN3-791 : *PUP: il prend ses animaux [*] et son animal

*PUP: et euh@i euh@i ja@nld [= oui] était [*] à la maison

f. NN3-790 : <een hert>@nld [= un cerf] \# et \# il euh@i il évait [*] une [/] une [*] grande [*] animal

\subsubsection{L'imperfectif apparaît chez les apprenants FL2 après le perfectif}

L'une des quatre affirmations spécifiques de l'hypothèse aspectuelle d'Andersen prédit que l'imperfectif apparaît après le perfectif dans l'interlangue de l'apprenant L2. Les résultats présentés dans le tableau 2 corroborent l'assertion étant donné que les apprenants NN1 ne produisent que des passés composés, essentiellement utilisés selon nos analyses pour marquer le perfectif, et que les premières occurrences d'imparfaits, la conjugaison qui indique l'imperfectif, sont produites plus tard par les apprenants NN3. 


\subsubsection{Les apprenants FL2 marquent d'abord le perfectif avec des verbes téliques}

Le deuxième volet de l'hypothèse aspectuelle rattache l'expression de l'aspect à certaines classes sémantiques de verbes (aspect lexical). Il prédit que les apprenants marquent en premier lieu le perfectif avec des verbes téliques avant de marquer celui des verbes atéliques.

Cinq des sept passés composés produits dans le corpus NN1 proviennent effectivement de trois verbes téliques (ex. 13), mais les deux autres verbes sont atéliques (ex. 14).

(13) a. NN1-4 : le matin le [/] le petit garçon \# voit le [*] grenouille $\underline{\text { est \# partir [*] }}$

b. NN1-227 : <ils sont> [//] \#\# ils voient euh@i le [*] grenouille est parti.

c. NN1-227 : grenouille euh@i il [*] est parti

d. NN1-227 : il est tombE

e. NN1-141 : la grenouille que cherchE [//] euh@i ils cherchE [//] euh@i ont trouvE

(14) a. NN1-176 : le chien et le garçon $\underline{a}$ regardE $[*]$ pour [*] le [*] grenouille

b. NN1-128 : l'enfant $\underline{\boldsymbol{a} \text { chant } \boldsymbol{E}}$

La grande majorité des passés composés produits dans le corpus NN3 sont également des verbes téliques (83\%, ex. 15).

(15) a. NN3-790 : le [*] grenouille est parti

b. NN3-790 : mais le chien $\underline{\text { a frappé }}\left[{ }^{*}\right]$ le [//] \# l'arbre et les abeilles $\underline{\text { sont tombées }}$

c. NN3-795 : le garçon est tombé

d. NN3-796 : il [/] il $\underline{\text { a entendu }}$ des bruits

Les données du corpus tendent donc à confirmer cette assertion du deuxième volet de l'hypothèse aspectuelle.

\subsubsection{L'imperfectif apparaît d'abord avec des verbes atéliques}

L'hypothèse aspectuelle prédit que l'imperfectif est produit dans un premier temps pour les verbes atéliques et seulement par après pour les verbes téliques.

Le corpus NN3 contient neuf occurrences de l'imparfait, dont sept sont atéliques (était cherchE, avait, regardait et deux occurrences de était et cherchait) et une est télique (était attaqué). La classe sémantique de la dernière occurrence ne peut être identifiée étant donné qu'il s'agit d'une forme ininterprétable, mais où le suffixe de l'imparfait est identifié (évait). Il est cependant important de remarquer que le seul verbe télique est construit à la voix passive et que la flexion de l'imparfait est donc marquée sur l'auxiliaire être. La fréquence relative des verbes téliques conjugués à l'imparfait augmente légèrement dans le corpus NN6 (17\%, cf. ex. 16), et la grande majorité des imparfaits produits par les apprenants NN6 sont toujours des verbes atéliques (cf. ex. 17).

(16) a. NN6-Sn13 : *PUP: il y avait une fois un garçon et son petit chien Pluto

*PUP: qui avait attrapé une grenouille

*PUP: il l'appelait Fien

*PUP: ah non c'est pas un nom français Fien

b. NN6-Me20 : *PUP: mais ils ne le [*] trouvaient pas

*PUP: alors ils décidaient d'aller le [*] chercher

c. NN6-Me20 : et alors uhm le cerf élevait la tête

(17) a. NN6-Le17 : le chien va avec son tête dans le pot où le grenouille était chaque chaque soir

b. NN6-Me20 : il tenait des cornes d'un cerf 


\section{c. NN6-628 : et euh@i le lendemain le [/] euh@i le petit gamin il a vu que le [*] grenouille n'était pas là}

Les résultats des études de Bartning et Schlyter (2004) et de Kihlstedt (2002) indiquent que l'imperfectif est d'abord marqué auprès des verbes être et avoir et seulement par après pour les verbes lexicaux. Les données décrites ci-dessus confirment partiellement leurs résultats dans la mesure où les premiers imparfaits produits comprennent déjà quelques verbes lexicaux (cf. ex. 11b-c, 12b-d).

\section{Commentaires et conclusions}

La contribution a permis d'examiner les développements de l'appariement entre les conjugaisons du passé et l'indication du temps ou de l'aspect dans l'interlangue d'apprenants de FL2. Pour ce faire, les données issues des productions orales des apprenants ont été confrontées aux différents volets de l'hypothèse aspectuelle d'Andersen.

Selon le premier volet de l'hypothèse, les premières formes des conjugaisons du passé qui sont produites marquent l'aspect et non le temps. Les seules formes des conjugaisons du passé produites par les apprenants NN1 sont des passés composés et leur analyse indique qu'elles semblent plutôt marquer une nuance aspectuelle, à travers l'opposition de formes verbales simples et composées ou par le caractère fortement télique du lexème verbal convoqué. Les premières formes verbales qui apportent une nuance temporelle apparaissent seulement chez les apprenants NN3. Les données de notre corpus semblent donc confirmer la primauté de l'aspect sur le temps dans le développement de l'appariement forme - fonction auprès des conjugaisons du passé.

L’imperfectif apparaît après le perfectif affirme un deuxième volet de l'hypothèse. Les données confirment également cette première assertion spécifique, étant donné que la forme qui marque l'imperfectif, l'imparfait, n'apparaît qu'à partir du corpus NN3, tandis que les apprenants NN1 produisent déjà des passés composés, qui marquent le perfectif.

L'hypothèse prédit ensuite l'ordre développemental du perfectif et de l'imperfectif en fonction de l'aspect lexical inhérent des lexèmes verbaux convoqués. Le perfectif serait en premier lieu utilisé avec les verbes téliques avant d'être utilisé avec les verbes atéliques. L’imperfectif apparaîtrait d'abord avec des verbes atéliques et ensuite seulement avec les verbes téliques. Les données de l'étude ne permettent pas de confirmer avec certitude l'emploi premier du perfectif avec des verbes téliques, étant donné que les apprenants les moins avancés utilisent déjà des verbes téliques et atéliques conjugués au passé composé. Leur nette prédominance dans la production du groupe NN1 tend néanmoins à indiquer la primauté de la combinaison passé composé/ verbe télique au début de l'exploitation du perfectif. Par contre, les données tendent à corroborer plus nettement la primauté de l'imperfectif avec des verbes atéliques. L'importance de l'aspect lexical inhérent des verbes dans le surgissement des premières occurrences des perfectifs et des imperfectifs semble donc modérément confirmée.

Les données de notre corpus confirment donc prudemment l’importance et la primauté du marquage de l'aspect dans l'interlangue des apprenants FL2. Cette primauté de l'aspect permet sans doute aussi de comprendre la primauté des formes du passé composé, qui s’opposent par l'aspect accompli au présent qui indique l'accomplissement. Le surgissement de l'imparfait semble aller de pair avec la prise en compte de l'expression du temps passé par le passé composé et donc aussi de la nécessité d'introduire une dimension d'accomplissement au passé. L'analyse détaillée des lexèmes verbaux convoqués indique que leur aspect lexical inhérent semble aussi jouer un rôle dans l'ordre développemental des conjuguaisons du passé, bien que certaines affirmations de l'hypothèse aspectuelle d'Andersen n'aient pas pu être pleinement confirmées. Les résultats décrits pourront être complétés et précisés par l'analyse de l'emploi des coverbes aspectuels (sous presse) et par la comparaison avec les productions de natifs. 


\section{Références bibliographiques}

Andersen, R. W. (1986). El desarrollo de la morfologia verbal en el español como segundo idioma. Adquisición de lenguaje/Aquisição da linguagem. Frankfurt : Vervuert. 115- 138.

Andersen, R. W. (1991). Developmental sequences : the emergence of aspect marking in second language acquisition. Crosscurrents in second language acquisition and linguistic theories. Amsterdam : John Benjamins. 305-324.

Ayoun, D. (2001). The role of negative and positive feedback in the second language acquisition of passé composé and imparfait. Modern Language Journal, 85, 226-243.

Ayoun, D. (2004). The Effectiveness of Written Recasts in the Second Language Acquisition of Aspectual Distinctions in French : A Follow-Up Study. The Modern Language Journal, 88, 31-55.

Ayoun, D. (2005). The acquisition of tense and aspect in L2 French from a Universal Grammar perspective. Tense and Aspect in Romance Languages : Theoretical and applied perspectives. Amsterdam : John Benjamins. 79-127.

Bardovi-Harlig, K. (1994). Anecdote or evidence ? Evaluating support for hypotheses concerning the development of tense and aspect. Research methodology in second language acquisition. Hillsdale : Erlbaum. 243-282.

Bardovi-Harlig, K. (2000). Tense and Aspect in Second Language Acquisition : Form, Meaning, and Use. Malden : Blackwell Publishers.

Bartning, I., Schlyter, S. (2004). Itinéraires acquisitionnels et stades de développement en français. Journal of French Language Studies, 14, 281-299.

Guillaume, G. (1964). Langage et science du langage. Paris-Québec : Nizet-Presses de Laval.

Howard, M. (2002). L'acquisition des temps du passé en français par l'apprenant dit avancé. Les temps du passé français et leur enseignement. Amsterdam-New York : Cahiers Chronos. 181-204.

Kaplan, M. A. (1987). Developmental patterns of past tense acquisition among foreign language learners of French. Foreign language learning : a research perspective. Cambridge, MA : Newbury House. 52-60.

Kihlstedt, M. (1998). La référence au passé dans le dialogue. Étude de l'acquisition de la temporalité chez des apprenants dits avancés de français. Cahier de la recherche 6. Thèse de doctorat, Université de Stockholm.

Kihlstedt, M. (2002). Reference to past events in dialogue. The L2 Acquisition of Tense-Aspect Morphology. Amsterdam : John Benjamins. 323-361.

Klein, W. (1994). Time in language. London : Routledge.

Klein, W. (1995). Frame of analysis. The acquisition of temporality in a second language. Amsterdam : John Benjamins. 17-29.

Li, P., Shirai, Y. (2000). The Acquisition of Lexical and Grammatical Aspect. Berlin : Mouton de Gruyter.

MacWhinney, B. (2000). The CHILDES Project : Tools for Analyzing Talk. $3^{\text {rd }}$ edition. Mahwah, NJ : Lawrence Erlbaum.

Mayer, M. (1969). Frog, where are you? New-York : Dial.

Noyau, C. (1997). Processus de grammaticalisation dans l'acquisition de langues étrangères: la morphologie temporelle. L’acquisition de la syntaxe. Besançon : Annales littéraires de l’Université de Franche-Comté. 223252.

Noyau, C., Houdaifa, E.-T., Vasseur, M.-T., Véronique, D. (1995). The acquisition of French. The acquisition of temporality in second language. Amsterdam : John Benjamins. 145-209.

Perdue, C., Benazzo, S., Giuliano, P. (2002). When finiteness gets marked : the relation between morphosyntactic development and use of scopal items in adult language acquisition. Linguistics, 40 (4), 849-890.

Perdue, C., Klein, W. (1992). Why does the production of some learners not grammaticalize ? Studies in Second Language Acquisition, 14, 259-272.

Reichenbach, H. (1947). The Tenses of Verbs. Elements of Symbolic Logic. New York : The Macmillan Company.

Riegel, M., Pellat, J.-Ch., Rioul, R. (2009). Grammaire méthodique du français. Paris : Presses Universitaires de 
France.

Salaberry, R. (1999). The development of past tense verbal morphology in classroom L2 Spanish. Applied Linguistics, 20, 151-178.

Schlyter, S. (2003). Stades de développement en français L2 : Exemples d’apprenants suédophones, guidés et nonguidés, du 'Corpus Lund'. Manuscrit, Université de Lund.

http://lup.lub.lu.se/luur/download?func=downloadFile\&recordOId=526759\&fileOId=624010

Starren, M. (2001). The Second Time : The Acquisition of Temporality in Dutch and French as a Second Language. Utrecht : LOT.

Ullman, M. (2001). The Declarative/Procedural Model of Lexicon and Grammar. Journal of Psycholinguistic Research, 30 (1), 37-69.

Vendler, ,Z. (1957). Verbs and Times. The Philosophical Review, 66 (2), 143-160.

Vendler, Z. (1967). Linguistics in Philosophy. Ithaca: Cornell University Press.

Véronique, D. (2009). L'acquisition de la grammaire du français, langue étrangère. Paris : Didier.

Wilmet, M. (2010). Grammaire critique du français. $5^{\text {ème }}$ édition. Bruxelles: Be Boeck-Duculot.

\footnotetext{
${ }^{1}$ La tradition scolaire française distingue deux auxiliaires (avoir et être) et deux semi-auxiliaires (aller et venir de) pour exprimer le temps ou l'aspect et cinq auxiliaires de mode (devoir, falloir, pouvoir, savoir et vouloir). Le caractère vague de cette catégorisation, basée sur le sens, a fréquemment été dénoncé. Voici pourquoi Wilmet (2010) propose de seulement distinguer les auxiliaires être et avoir et les « coverbes » d'aspect et de modalité.
} 